\title{
Surfaces and Their Interfaces Meet Biology at the Bio-interface
}

\author{
CANDAN TAMERLER ${ }^{1,2}$ \\ 1.--Mechanical Engineering Department and Bioengineering Research Center (BERC), University \\ of Kansas, 1530 W, 15th St. Learned Hall, Lawrence, KS 66045, USA. 2.-e-mail: ctamerler@ \\ ku.edu
}

Surfaces and bio-interfaces have undoubtedly become a topic which signifies one of the most rapidly expanding fields that is innovative and dynamic across the disciplines from engineering to life sciences. All solid material systems have boundaries, of which the properties are different from bulk material at the nanoscale. How these "inbetween regions" merge into one another becomes a critical challenge, and also a fascinating question which has moved to the forefront in the development of new technologies relevant to all aspects of life, from biomedical to microelectronics to energy production.

Biological materials exhibit complex structureproperty relationships which exist at multiplelength scales with elegant hierarchical organization. Understanding the interface of native biological materials and harnessing these design strategies to develop biomimetic, bioinspired, and bio-enabled materials will expedite the accomplishing of functionally integrated materials. An indepth understanding of the interface remains critical in the design of the bio-interface. The design also becomes essential to developing the controlled and predictable interactions on the surfaces, a reciprocal relationship unique to the bio-interface. Surfaces and bio-interfaces are the vital components of all bio-related materials, processes, and devices which span areas as diverse as bioinspired materials, including optics and energy conversion, regenerative and restorative medicine, biosensors, diagnostics, therapeutics, and smart textiles.

The papers that are selected for this "Surfaces and Bio-interfaces" topic of JOM capture a collection of original research papers, as well as reviews, from a distinguished group of scientists covering the synthesis, characterization, modeling and specific applications for different materials and their interest areas. The scientific challenges and opportunities are continuing to push the boundaries of our collective understanding of surfaces and bio-interfaces, and thus are bringing new ways of looking at the interface to explore the interactions between biological components and surfaces, and to understand and to develop novel bio-inspired, biomimetic, and bio-enabled materials and processes. Articles have been selected from these gifted investigators in order to bridge our fundamental understanding of soft and hard biomaterial interfaces and to bring into focus the applications which include biomedical product development and nano- to micro-fabrication systems. Contributions to the topic were published in Surfaces and Bio-interfaces: Part I in April 2015 (JOM vol. 67, no. 4), and Surfaces and Bio-interfaces: Part II in this issue. Following is a brief introduction to these papers. Publication details are in the references.

The importance of biomolecule self-assembly on the solid materials has become increasingly recognized. Molecular recognition plays a critical role in biological interactions; hierarchical composite structures often found in biological materials are a result of molecular recognition where biomolecules, mainly proteins, play a crucial role. To explore this topic, we have articles which emphasize the role of peptides as the biological self-assemblers towards designing bio-materials interfaces. In designing biohybrid functional materials and surfaces, material selectivity is vital and peptides have come a long way; however, there is still a long way to go. There is a growing interest in applying peptides as materials-selective assemblers and self-organizers, but considering the boundless application opportunities of peptides in molecular technologies, their utility is still based largely on empirical understanding of solid surface binding characteristics.

Adams and coworkers ${ }^{1}$ demonstrated a self-organized cell templating directed by a gold surfaceselective peptide interface. Controlled cell filamentation is a step towards next-generation living material interfaces. Peptides binding to gold surfaces were also investigated in Corni's paper, in which he extends this analysis to the interplay between geometrical matching of peptide-surface features as a function of the structural flexibility. ${ }^{2}$ Tucker and coworkers ${ }^{3}$ investigated the binding of 
histidine-tagged F1-ATPase motor protein to gold surface towards designing motor protein-based nanobiodevices. Yucesoy et al. presented a chimeric peptide design approach to create an antimicrobial implant material surface where the engineered peptide has an anchoring site specific to titanium alloy surface as well as an antimicrobial domain, both simultaneously presented at the bio-nano interface. $^{4}$

Topoleski et al. ${ }^{5}$ present a comprehensive review on the subject of biomaterials currently used for artificial joints. The authors discuss the surface properties and summarize advancement on surface modifications which are introduced at the bearing interface to improve their wear properties. They also provide an extensive summary on the wear performance of orthopedic articulating couples that are commercially available, extending the summary to new materials which are attracting interest in total joint replacement like cobalt-chromium-molybdenum (CoCrMo) and Ti6Al4V alloys. Another surface treatment study on Ti6Al4V orthopedic grade alloy is described by Fuerst et al. ${ }^{6}$ where they apply titanium-tantalum coatings to increase wear resistance and improve performance of Ti-alloys. Shokuhfa and coworkers $^{7}$ focus on the interfacial stress generated by the Ti6Al4V rods when they are inserted into a simulated bone. They describe the mechanical stability of biomedical Ti6Al4V rods aligned with nanotube structures.

Nanoclay composites are attracting interest as potential scaffolds for bone tissue engineering. They are introduced to improve the mechanical properties of the scaffolds and generate a mineralized extracellular matrix similar to bone. Katti et al. ${ }^{8}$ present a molecular dynamics simulation approach for understanding the effect of nanoclays on nanocomposites. Simulation studies provide a detailed analysis on the molecular interactions between the different constituents where nanoclays act as the modifiers. A paper on tissue engineering application is presented by Ciobanu et al., ${ }^{9}$ in which bismuth substitution for calcium in hydroxyapatite nanopowders is described using a wet chemical method. They highlight the antimicrobial property of bismuth in the resulting nanopowders.

The next set of papers focuses on teeth, another example of heterogeneous, hierarchical and composite structure. The first paper in this set is on polymeric composite restoratives which have become an alternative to amalgam-based restorative materials due to many properties including the ability to bond to both dentin and enamel. Spencer and coworkers ${ }^{10}$ describe a new initiator system which offers a high degree of polymer conversion and therefore prevents potential leaching of unreacted compounds. This is a common concern for dimethacrylate-monomers-based matrix formation which is predominant in polymer-based composites. The developed initiator system may not only prevent the local and systemic toxicity but also provide extended aging. In dental metallic materials, Curteanu and coworkers ${ }^{11}$ investigate the corrosion resistance of titanium, $\mathrm{NiTi}$ and $\mathrm{NiTi}$ alloys in artificial saliva and develop an adaptive regression model building upon experimental data as a predictive tool.

The next paper by M.L. Snead focuses on the cellular and molecular basis for enamel formation providing a perspective towards enamel generation using molecular technologies. ${ }^{12}$ Using a knock-in genetic strategy in mice models, Snead summarizes how cell-to-protein matrix interactions dominate enamel formation. These interactions become very critical in the soft phase of early enamel development where defects in the amelogenin-rich matrix result in mineral defects.

Zustiak and coworkers summarize how biomaterials properties can be critical for cancer stem cell growth, an area which has recently been highly recognized. ${ }^{13}$ Their results suggest that the defined interfaces which are introduced during the growth on the selected biomaterial have a profound effect on expressing the characteristics of cancer stem cells.

Surface chemistry of nanoparticles determine their fate in many of the biological applications. Webster and coworkers describe the effect of dextran coating on cerium oxide nanoparticles for bone cancer cells. ${ }^{14}$ Their results suggest polymer-based surface coatings may be used to tune their beneficial antioxidant property to protect healthy cells from free radicals. Similarly, polymer nanospheres are attracting interest as drug delivery vehicles due to their high surface area and low density characteristics. Edirisinghe and coworkers ${ }^{15}$ describe the effect of solvent and temperature on the size and surface features of nanospheres which are produced by an engineered microfluidic system. Surface roughness is shown to compensate for size difference building upon the release studies performed using a dye molecule.

Carbon-based materials have been widely utilized due to their intrinsic properties such as high surface area, mechanical strength and chemical and thermal stabilities. Saito and coworkers ${ }^{16}$ present solution plasma processing as a viable source to control the structure in carbon nanomaterials in which the synthesized materials can be designed with the desired properties using low input energy and temperature, and expanding their utilization from energy storage systems to biosensors. In fact, Akceoglu et al. ${ }^{17}$ demonstrate that graphite-oxidecellulose composites can be used as solid-phase genomic DNA extraction agents with a high extraction efficiency compared to conventional methods. These DNA/carbon hybrid composite materials may possibly serve as new-generation extraction kits.

The functional aspects of plant surface structures present diverse models for biomimetics materials, as successfully done for lotus leaves. More than 400 
million years of evolution offer endless examples for the generation of biomimetic materials with functional surface structures. Among these properties, wettability, such as super-hydrophobicity and super-hydrophilicity, has been extensively investigated, and these structures have been an inspiration for developing various nanostructured materials with functional surfaces and coatings. Carnivorous plants have attracted interest due to their various trapping systems and strategies. Po$\mathrm{Yu}$ Chen and coworkers ${ }^{18}$ propose how the wetness transition is achieved under natural environment for the pitcher plant Nepenthes, a phenomenon still not fully understood.

Surfaces and interfaces play an important role in designing both structural and functional materials, and controlling their performances across multiple length scales and bio-interfaces is one of the most dynamic and expanding area in this field. Bio-interfaces are vital in designing the functionality of bio-related processes and devices in diverse areas including biotechnology, biosensors, stem cell technology, diagnostics, therapeutics, biomimetic materials, additive biomanufacturing, regenerative medicine, and biomaterials for medical implants and functional tissue engineering. I trust you will find inspiration from the collected manuscripts.

\section{REFERENCES}

1. B.L. Adams, M.M. Harley, J.P. Jahnke, and D.N. StratisCullum, JOM, 67 (2015). doi:10.1007/s11837-015-1662-7.

2. S. Corni, JOM 67, 781 (2015).

3. J.K. Tucker, M.L. Richter, and C.L. Berrie, JOM (2015). doi:10.1007/s11837-015-1378-8.

4. D.T. Yucesoy, M. Hnilova, K. Boone, P.M. Arnold, M.L. Snead, and C. Tamerler, JOM 67, 733 (2015).

5. S.J.L. Sullivan and L.D. Timmie Topoleski, JOM (2015). doi:10.1007/s11837-015-1543-0.

6. J. Fuerst, D. Medlin, M. Carter, J. Sears, and G.V. Voort, JOM 67, 775 (2015).

7. S. Patel, G.F. Solitro, C. Sukotjo, C. Takoudis, M. Mathew, F. Amirouche, and T. Shokuhfar, JOM (2015). doi:10.1007/ s11837-015-1341-8.

8. S. Sharma, K.S. Payne, and D.R. Katti, Katti. JOM 67, 733 (2015).

9. G. Ciobanu, A.M. Bargan, and C. Luca, JOM (2015). doi:10. 1007/s11837-015-1467-8.

10. R. Chelariu, G.D. Suditu, D. Mareci, G. Bolat, N. Cimpoesu, F. Leon, and S. Curteanu, JOM 67, 767 (2015).

11. X.P. Ge, Q. Ye, L.Y. Song, J.S. Laurence, and P. Spencer, JOM 67, 796 (2015).

12. M.L. Snead, JOM 67, 788 (2015).

13. F. Ordikhani, K. Yonghyun, and S. Zustiak, JOM (2015). doi:10.1007/s11837-015-1626-y.

14. H. Yazici, E. Alpaslan, and T.J. Webster, JOM 67, 804 (2015).

15. I. Kucuk and M. Edirisinghe, JOM 67, 811 (2015).

16. L. Hoonseung, U. Tomonaga, and N. Saito, JOM (2015). doi:10.1007/s11837-015-1660-9.

17. G.A. Akceoglu, O.L. Lin, and N. Saito, JOM (2015). doi:10. 1007/s11837-015-1610-6.

18. C.P. Hsu, Y.M. Lin, and P.Y. Chen, JOM 67, 744 (2015). 